\title{
Dysregulated chaperones associated with cell proliferation and negative apoptosis regulation in the uterine leiomyoma
}

\author{
BLENDI URA $^{1}$, FEDERICA SCRIMIN ${ }^{1}$, GIORGIO ARRIGONI ${ }^{2,3}$, \\ MICHELANGELO ALOISIO ${ }^{1}$, LORENZO MONASTA ${ }^{1}$ and GIUSEPPE RICCI ${ }^{1,4}$ \\ ${ }^{1}$ Institute for Maternal and Child Health-IRCCS ‘Burlo Garofolo', I-34137 Trieste; ${ }^{2}$ Department of \\ Biomedical Sciences, University of Padua, I-35122 Padua; ${ }^{3}$ Proteomics Center, University of Padua, I-35129 Padua; \\ ${ }^{4}$ Department of Medical, Surgery and Health Sciences, University of Trieste, I-34128 Trieste, Italy
}

Received March 15, 2017; Accepted December 11, 2017

DOI: $10.3892 / \mathrm{ol} .2018 .8325$

\begin{abstract}
Uterine leiomyomas are benign smooth muscle cell tumors that originate from the myometrium. In this study we focus on dysregulated chaperones associated with cell proliferation and apoptosis. Paired tissue samples of 15 leiomyomas and adjacent myometria were obtained and analyzed by two-dimensional gel electrophoresis (2-DE). Mass spectrometry was used for protein identification and western blotting for 2-DE data validation. The values of 6 chaperones were found to be significantly different in the leiomyoma when compared with the myometrium. A total of 4 proteins were upregulated in the leiomyoma and 2 proteins were downregulated. Calreticulin and $78 \mathrm{kDa}$ glucose-regulated protein were further validated by western blotting because the first is considered a marker of cell proliferation, while the second protects against apoptotic cell death. In addition, we also validated the two downregulated proteins heat shock protein $\beta-1$ and heat shock $70 \mathrm{kDa}$ protein 1A. Our study shows the existence of a dysregulation of chaperone proteins associated with leiomyoma development. Functional studies are needed to ascertain the role of these chaperones in the leiomyoma. This may be crucial for the further development of specific inhibitors against the activity of these proteins in order to block the growth of the leiomyoma.
\end{abstract}

\section{Introduction}

Uterine leiomyomas are benign smooth muscle cell tumors originating from the myometrium. These tumors can occur in $70-80 \%$ of women and are the first gynecological reason why women undergo hysterectomy (1). It has been ascertained that leiomyoma growth is strongly dependent on steroids, growth

Correspondence to: Dr Blendi Ura, Institute for Maternal and Child Health-IRCCS 'Burlo Garofolo', 65/1 Via dell'Istria, I-34137 Trieste, Italy

E-mail: blendi.ura2006@libero.it

Key words: proteomics, leiomyoma, molecular chaperones, proteins, two-dimensional electrophoresis factors, transforming growth factor- $\beta$ (TGF- $\beta$ )/Smad, wingless-type (Wnt)/ $\beta$-catenin, retinoic acid, and vitamin D (2). We also established that protein expression is associated hormone secretion (3).

Molecular evidence supports a role for genes, involved in extracellular matrix (ECM) production, in tumor volume expansion (4). Genetic alterations are considered insufficient drivers of tumor development, and additional complex signaling pathway (p38 MAPK pathway, VEGF signaling pathway, UPR pathway) alterations may be crucial (2). Heat shock proteins have often been associated with tumor progression through their ability to evade apoptosis and senescence (5). HSP90 plays an important role in estradiol signaling, chaperoned unbound estrogen receptors (ER). This protein helps in molecular trafficking inside the nucleus, leading the modulation of the transcriptional activities $(6,7)$ of target genes correlated with tumor development.

Several external factors, including proteins, can induce alterations in the mechanotransduction signal from ECM via the transmembrane (8) receptor to the interior of the cell, leading to cell migration (9), metabolic dysregulation (10), proliferation and growth.

The objective of our study was to individuate dysregulated chaperonins with possible involvement in leiomyoma pathobiology.

\section{Materials and methods}

General. Tissue samples were obtained from 15 premenopausal patients who underwent hysterectomy for symptomatic uterine leiomyomas. All procedures conformed with the Declaration of Helsinki and were approved by the Review Board of the Institute for Maternal and Child Health-IRCCS 'Burlo Garofolo' (Trieste, Italy). All involved subjects signed a written informed consent. The median age of patients was 45 years, with a minimum of 36 and a maximum of 48 years.

Tissue samples. Two samples were collected from each patient: One from the central area of the leiomyoma and one from the unaffected myometrium. All leiomyomas were confirmed histologically as benign ordinary leiomyomas. Samples were stored at $-80^{\circ} \mathrm{C}$ until proteomic analysis was performed. 
Two-dimensional gel electrophoresis (2-DE) and spot quantification. 2-DE analysis was performed as previously described (11). Briefly, clean samples of leiomyoma and myometrium (200 mg each) were homogenized in $1.2 \mathrm{ml}$ of dissolution TUC buffer [7 M urea, $2 \mathrm{M}$ thiourea, 4\% CHAPS, $40 \mathrm{mM}$ Tris, $65 \mathrm{mM}$ DTT and $0.24 \%$ Bio-Lyte (3-10)] with a protease inhibitor mix (2 mM PMSF, $1 \mathrm{mM}$ benzamidine, $1 \mathrm{mM}$ EDTA, $1 \mathrm{mM} \mathrm{NaF})$. After vortex, the solutions were centrifuged at $10,000 \mathrm{x} \mathrm{g}$ at $4^{\circ} \mathrm{C}$ for $30 \mathrm{~min}$ and the protein content of the supernatant was determined using the Bradford assay. For the 2-DE analysis, 1,000 $\mu \mathrm{g}$ of proteins from each sample were used. Isoelectric focusing (IEF) was performed by using NL IPG Readystrips, 18-cm pH 3-10 in a Protean IEF cell (Bio-Rad Laboratories, Inc., Hercules, CA, USA) set to $170,000 \mathrm{Vh}$. After IEF, the IPG strips were equilibrated for $20 \mathrm{~min}$ in equilibration buffer (6 M urea, 2\% SDS, 50 mM Tris- $\mathrm{HCl} \mathrm{pH} 8.8,30 \%$ glycerol, and 1\% DTT) and in equilibration buffer containing $4 \%$ iodoacetamide instead of DTT. For the second dimension, equilibrated IPG strips were transferred onto a $12 \%$ SDS-PAGE and were run on Protean II XL Cell (200 V constant voltage) until the bromophenol blue reached the bottom of the gel. After the second dimension, gels were stained with colloidal Coomassie Blue, and excess dye was removed with distilled water. Two experimental replicates were performed per sample.

Molecular masses were determined by precision protein standard markers (Bio-Rad Laboratories, Inc.). 2-DE gels were scanned with a Molecular Imager PharosFX system (Bio-Rad Laboratories, Inc.). The quantitative analysis of the spots was conducted out using the ProteomeWeaver 4 program (Bio-Rad Laboratories, Inc.).

Differences were considered to be significant when the ratio of the mean percentage relative volume (\%V=Vsingle spot/Vtotal spot) was 1.5-fold for upregulated and 0.6-fold for downregulated proteins, and satisfied the non-parametric Wilcoxon signed-rank test for matched samples $(\mathrm{P}<0.05)$. Fold-change was calculated as the ratio between the mean percentage relative volume of the uterine leiomyoma and the normal myometrium.

Trypsin digestion and MALDI analysis. Spots from 2-DE were digested with sequencing grade-modified trypsin (Promega Corporation, Madison, WI, USA) and analyzed by mass spectrometry (MS), as described by Ura et al (8).

The protein spots selected by 2-DE analysis were manually excised from the gels, washed with $50 \mathrm{mM} \mathrm{NH} 4 \mathrm{HCO} 3$ and acetonitrile (ACN). After drying, $10 \mu \mathrm{l}$ of trypsin $(12.5 \mathrm{ng} / \mu \mathrm{l}$ in $50 \mathrm{mM}$ NH4HCO3) were added to each gel piece and kept in ice for $30 \mathrm{~min}$ before being incubated overnight at $37^{\circ} \mathrm{C}$. Peptides were extracted from the gel pieces by three changes of $75 \% \mathrm{ACN} / 0.1 \%$ trifluoroacetic acid (TFA), dried under vacuum and then dissolved again in $10 \mu \mathrm{l}$ of $0.1 \%$ TFA. One $\mu \mathrm{l}$ of each sample was mixed with $1 \mu \mathrm{l}$ of (matrix $\alpha$-cyano-4-hydroxycinnamic acid, $5 \mathrm{mg} / \mathrm{ml}$ in $70 \% \mathrm{ACN} / 0.1 \%$ TFA) and $0.8 \mu \mathrm{l}$ of the final sample/matrix mixture were spotted onto a stainless steel MALDI target plate. Tandem mass spectrometry (MS/MS) analysis was performed on a MALDI-TOF/TOF 4800 mass spectrometer (AB Sciex, Framingham, MA, USA) in a data-dependent mode: A full MS scan was acquired, followed by MS/MS spectra of the 10 most intense signals.

Data were converted into Mascot generic format (MGF) files using the 4000 Series Explorer software (AB Sciex) and searched using Mascot search engine (version 2.4; Matrix Science, London, UK). Enzyme specificity was set to trypsin with one missed cleavage. Tolerance was set to $50 \mathrm{ppm}$ and $0.3 \mathrm{kDa}$ for precursor and fragment ions respectively, and carbamidomethylation of cysteine was set as fixed modification, while methionine oxidation was set as variable modification. Proteins were considered as positively identified if $\geq 2$ independent peptides were identified with $95 \%$ confidence.

String network and functional analysis. The different expression proteins thus identified were analyzed by STRING 10.0 (http://www.string-db.org/) for network generation and PANTHER 11.0 (Protein Analysis through Evolutionary Relationships; http:/www.pantherdb.org) and Gene Ontology (http://amigo.geneontology.org/rte). Proteins were then classified according to their involvement in biological processes and pathways.

Western blotting. Western blot analysis was performed as previously described (12). Briefly, an equal amount of protein $(30 \mu \mathrm{g})$ used for 2-DE analysis was separated by $12 \%$ SDS-PAGE and then transferred to a nitrocellulose membrane. After being saturated by $5 \%$ defatted milk, membranes were incubated overnight at $4^{\circ} \mathrm{C}$ with $1: 1,000$ diluted primary rabbit polyclonal antibody against CALR, with 1:200 diluted primary rabbit polyclonal antibody against HSPA5, with 1:300 diluted primary rabbit polyclonal antibody against HSPB1, and with 1:700 diluted primary rabbit polyclonal antibody against HSPA1A. After washing, membranes were incubated with a HRP-conjugated anti-rabbit IgG (Sigma-Aldrich; Merck KGaA, Darmstadt, Germany) in a dilution of 1:3,000. The protein signal was visualized using SuperSignal West Pico Chemiluminescent substrate (Thermo Fisher Scientific Inc., Ottawa, ON, Canada). The intensities of the immunostained bands were normalized with the total protein intensities measured by Coomassie blue from the same blot.

Statistical analyses. For paired samples of both 2-DE and western blot data, statistical analyses were carried out with the non-parametric Wilcoxon sign-rank test. $\mathrm{P}<0.05$ was considered to indicate a statistically significant difference. All statistical analyses were conducted with Stata/IC 14.2 for Windows (StataCorp LP, College Station, TX, USA).

\section{Results}

Proteomic studies. Comparative proteomic analysis was performed using 2-DE coupled with MS between uterine leiomyoma and myometrium tissues. Correlation analysis of gel-pairs performed well, with average matching efficiency of approximately $75 \%$. In this study, six protein spots belonging to chaperone proteins were found to be significantly dysregulated in leiomyoma samples if compared to the myometrium. Four spots were significantly upregulated ( $>1.5$-fold) and two were significantly downregulated $(<0.6$-fold) (Fig. 1). These spots 
Table I. Dysregulated chaperones identified by mass spectrometry in the leiomyoma and in the myometrium.

\begin{tabular}{|c|c|c|c|c|c|c|c|}
\hline $\begin{array}{l}\text { Accession } \\
\text { number }\end{array}$ & $\begin{array}{c}\text { Spot } \\
\text { number }\end{array}$ & $\begin{array}{c}\text { Protein } \\
\text { description }\end{array}$ & $\begin{array}{l}\text { Gene } \\
\text { symbol }\end{array}$ & $\begin{array}{l}\text { Sequence } \\
\text { coverage } \%\end{array}$ & $\begin{array}{l}\text { Fold- } \\
\text { change }^{\mathrm{a}}\end{array}$ & $\begin{array}{l}\text { Biological } \\
\text { function }\end{array}$ & Pathway \\
\hline P27797 & 2 & Calreticulin & CALR & 17.75 & 2.3 & $\begin{array}{l}\text { Positive regulation of } \\
\text { cell proliferation }\end{array}$ & UPR pathway \\
\hline P11021 & 3 & $\begin{array}{l}78 \mathrm{kDa} \text { glucose- } \\
\text { regulated protein }\end{array}$ & HSPA5 & 12.54 & 2 & $\begin{array}{l}\text { Negative regulation of } \\
\text { apoptotic signaling } \\
\text { pathway }\end{array}$ & $\begin{array}{l}\text { Apoptosis } \\
\text { signaling } \\
\text { pathway }\end{array}$ \\
\hline P30101 & 1 & $\begin{array}{l}\text { Protein disulfide- } \\
\text { isomerase A3 }\end{array}$ & PDIA3 & 7.22 & 1.9 & Protein folding & UPR pathway \\
\hline P55072 & 4 & $\begin{array}{l}\text { Transitional } \\
\text { endoplasmic reticulum } \\
\text { ATPase }\end{array}$ & $\mathrm{VCP}$ & 6.58 & 1.7 & $\begin{array}{l}\text { Flavin adenine } \\
\text { dinucleotide } \\
\text { metabolic process }\end{array}$ & UPR pathway \\
\hline E7EP94 & 5 & $\begin{array}{l}\text { Heat shock } 70 \mathrm{kDa} \\
\text { protein } 1 \mathrm{~A} / 1 \mathrm{~B}\end{array}$ & HSPA1A & 4.31 & 0.18 & $\begin{array}{l}\text { Protein complex } \\
\text { assembly }\end{array}$ & $\begin{array}{l}\text { Apoptosis } \\
\text { signaling } \\
\text { pathway }\end{array}$ \\
\hline P04792 & 6 & $\begin{array}{l}\text { Heat shock } \\
\text { protein } \beta-1\end{array}$ & HSPB1 & 12.20 & 0.02 & Protein folding & $\begin{array}{l}\text { p38 MAPK } \\
\text { pathway }\end{array}$ \\
\hline
\end{tabular}

${ }^{a}$ Fold-change was defined as the ratio of the mean $\% \mathrm{~V}$, according to the formula $\% \mathrm{~V}=(\mathrm{V}$ single spot $) /(\mathrm{Vtotal}$ spot $)$ of uterine leiomyoma and normal myometrium. UPR, unfolded protein response; MAPK, mitogen-activated protein kinase.
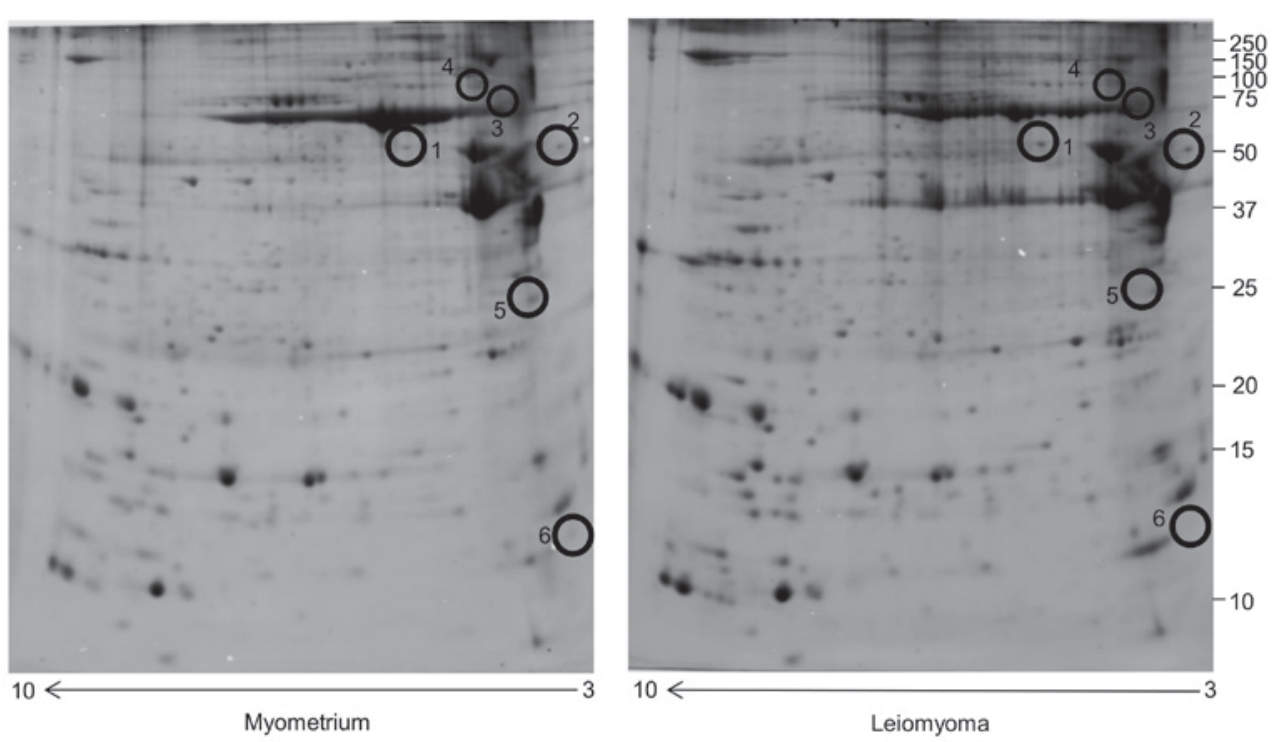

Figure 1. Two-dimensional electrophoresis map of normal myometrium and leiomyoma proteome. Immobilized pH gradient 3-10NL strips were used for the first dimension and $12 \%$ polyacrylamide gel was used for the second dimension. The numbered circles indicate the dysregulated chaperones.

corresponded to six proteins identified by MALDI-TOF/TOF (Table I). Spot quantification revealed that the four proteins significantly upregulated in the leiomyoma if compared to the myometrium were CALR, HSPA5, PDIA3 and VCP, while the two downregulated proteins corresponded to HSPA1A and HSPB1.

These proteins are involved in the promotion of cell proliferation (CALR), in the negative regulation of apoptosis (HSPA5), protein folding (PDIA3, HSPB1), flavin adenine dinucleotide metabolic process (VCP) and protein complex assembly (HSPA1A). PANTHER pathway analysis locates these proteins in several pathways (p38 MAPK pathway,
VEGF signaling pathway, UPR pathway) related to proliferation, migration and differentiation.

Immunohistochemical study of protein expression. In this study we validated the upregulated proteins CALR and HSPA5 because the first is considered a marker of cell proliferation, while the second protects against apoptotic cell death, and could be associated with the promotion of leiomyoma growth. In addition, we also validated the two downregulated proteins HSPB1 and HSPA1A. The expression of CALR, HSPA5, HSPB1 and HSPA1A in five leiomyomas was compared to the expression in matched normal myometrial tissue (previously 

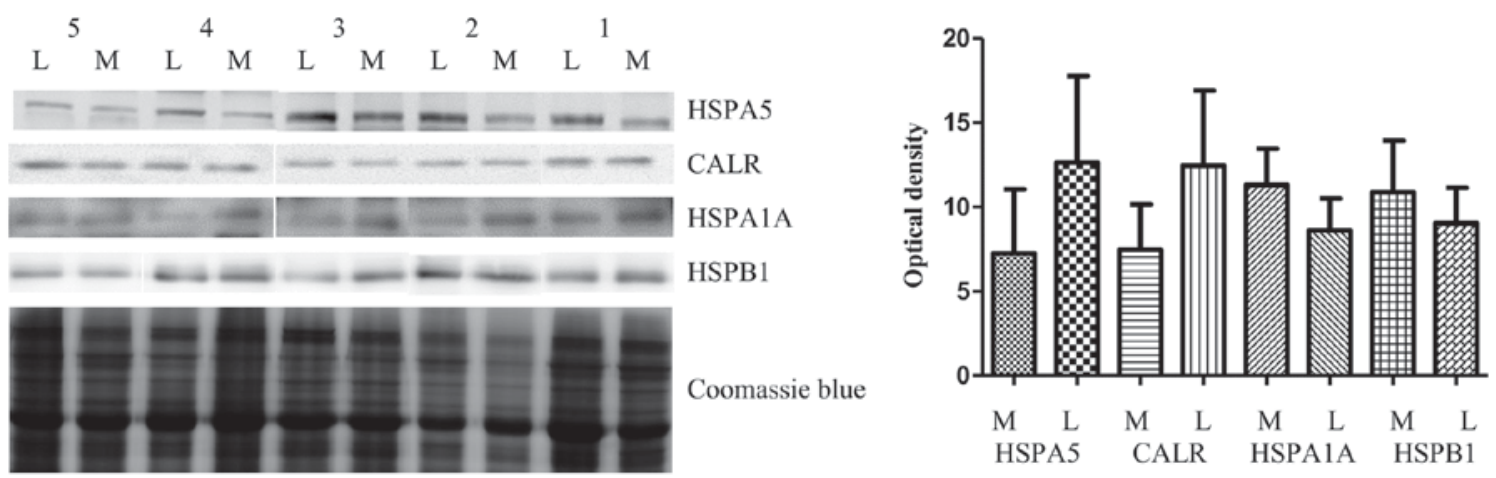

Figure 2. Western blot analysis of HSPA5 CALR, HSP1A1, HSPB1 in paired myometrium (M) and leiomyoma (L). The intensity of immunostained bands was normalized against the total protein intensities measured from the same blot stained with Coomassie Blue. Number 1-5 indicate the patients. The bar graph shows the relative expression (band density) of HSPA5, CALR, HSP1A1, HSPB1 in the myometrium and the leiomyoma. Results are shown as a histogram $(\mathrm{P}<0.05)$ and each bar represents mean \pm standard deviation.

A

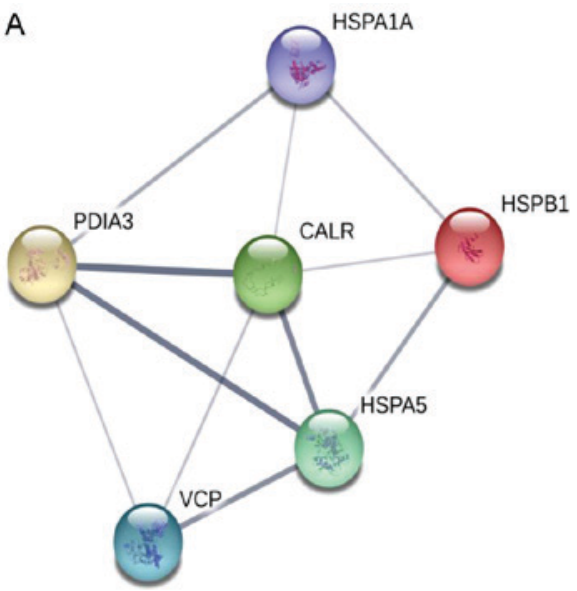

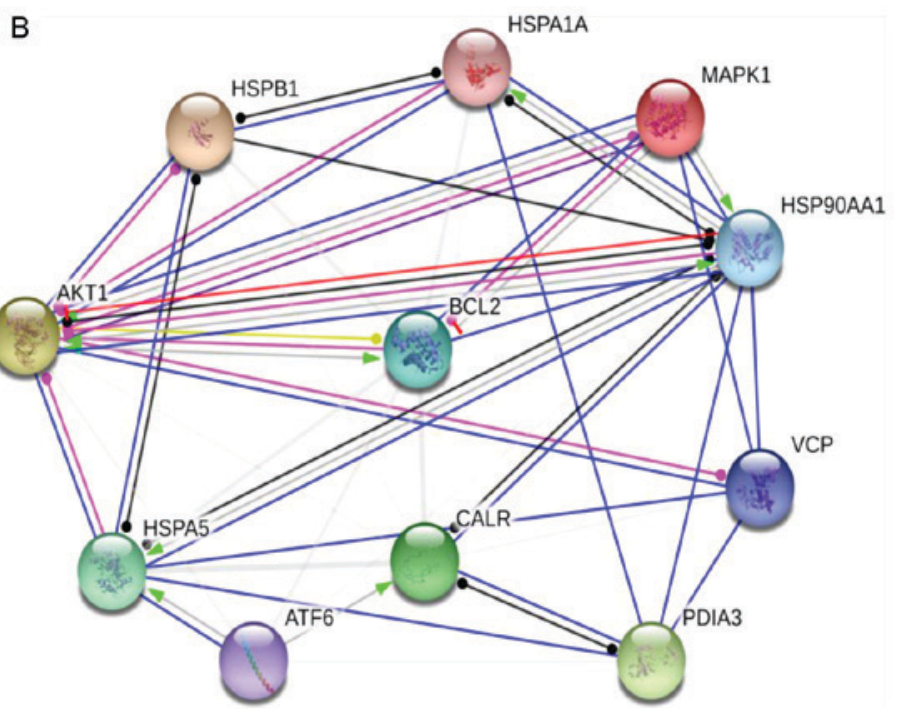

Figure 3. Prediction by STRING database. (A) Chaperones interaction on confidence prediction. (B) Chaperones and key proteins present in (p38 MAPK pathway, VEGF signaling pathway, UPR pathway) on molecular action prediction. For A, line thickness indicates the strength of data support. For B, line shape indicates the predicted mode of action, and colors indicate action types: Green, activation; red, inhibition; blue, binding; purple, catalysis; light blue, phenotype; fuchsia, posttranslational modification; black, reaction; yellow, transcriptional regulation.

used in 2-DE analyis) samples by western blot analysis (Fig. 2). The five patients shown in the figure are representative of the total 15 patients included in the study, based on both 2-DE and western blotting expression of CALR, HSPA5 (both always up regulated), and HPA1A and HSPB1 (both always downregulated).

CALR and HSPA5 expressions were significantly higher in the leiomyoma with respect to the myometrium, while HSPB1 and HSPA1A were significantly downregulated, confirming results obtained from the 2-DE analysis. As previously described, for the normalization of immunostained bands, we used the total protein intensities because the two major gene housekeeping $\beta$-actin and tubulin were upregulated (9).

Protein-protein interaction analysis. The chaperone proteins identified in this study were loaded to the STRING 10.0 software for protein-protein interaction network based on confidence and molecular action prediction. The strongest interaction were between: CALR and PDIA3 (combined score,
0.999), CALR and HSPA5 (combined score, 0.86), PDA3 and HSPA5 (combined score, 0.955), VCP and HSPA5 (combined score, 0.798) (Fig. 3A).

We further analyzed the interaction between MAPK1, BCL-2, AKT, ATF6, HSP90 (key proteins present in p38 MAPK pathway, VEGF signaling pathway, UPR pathway) and the proteins identified in our study. An interesting result based on action prediction was the activation of CALR, HSPA5 by ATF and HSPA1A, HSPA5 by HSP90. Finally, the software predicted the post-translational modification of HSPB1 by AKT1 (Fig. 3B).

\section{Discussion}

Chaperones may play a key role in promoting cell survival (13). Several study have established that chaperone proteins play a fundamental role in the molecular mechanisms that lead to the emergence and progression of the tumor $(14,15)$. At present it is clear that, within the tumor microenvironment, 
the increasing of chaperone activity induces a survival advantage on transformed cells over normal non-transformed cells (16).

To our knowledge, for the first time in this proteomics study we gathered data on the expression of several chaperones involved in cell proliferation and negative apoptosis regulation, and we put them in relation with several key pathways of tumor development.

2-DE is a powerful method for quantitative comparative proteomic studies, capable of simultaneous resolution of thousands of proteins, including isoforms or protein PTMs. Indeed, 2-DE has been proven to have a quite high reproducibility even across different laboratories (17) and even below the cutoff of 1.5 -fold-change that was used in our study.

Leemer and colleagues (18) conducted an in-depth study on the proteomic profile of leiomyoma and myometrium samples by label-free GeLC-MS/MS, resulting in the identification and intensity-based label-free quantification of more than 7000 proteins. We compared our data with theirs. The expression of PDIA3, HSPA5, CALR, was confirmed to be upregulated, while the expression of HSPA1A and HSPB1 was confirmed to be downregulated. The abundance of VLC was confirmed to be upregulated in the leiomyoma interstitial fluid (8).

Calreticulin is a calcium-binding chaperone that promotes folding, oligomeric assembly and quality control in the endoplasmic reticulum (ER), promoting cell growth and proliferation in tumor prostate (19). Calreticulin overexpression is mediated via the activation of the ATF6 pathway in myeloid leukemic cells (20).

Overexpression of calreticulin promotes cell proliferation, inducing the upregulation of VEGF. This protein binds to specific receptors, activating signal pathways with a key role in cell proliferation, survival, migration, like MAPK (mitogen-activated protein kinase) and ERK (Ras/extracellular signal regulated kinase) (21). This process could also be happening in the leiomyoma, where the overexpression of VEGF occurs (21).

HSPA5 is another calcium-binding chaperone playing an important role in maintaining cell viability against several kinds of stresses, including depletion of calcium from the endoplasmic reticulum (22). The overexpression of this protein in several tumors is induced by unfolded protein response.

HSPA5 protects tumor cells against apoptosis through various mechanisms: i) fighting protein aggregation in the ER; ii) binding $\mathrm{Ca}^{2+}$ and thus preventing calcium signaling in the cytosol; and iii) preventing the activation of pro-apoptotic components, such as BIK, BAX, pro-caspase 7 and pro-caspase 12 (23).

Pressinotti et al confirmed the role of PDIA3 as a protein involved in the positive regulation of apoptosis in prostate cancer (24).

In this study we found HSPA1A and HSPB1 to be downregulated in the leiomyoma if compared to the myometrium. In our previous study (8) we identified HSPA1A as less abundant in the leiomyoma interstitial fluid, this being in line with our present data.

HSPB1 belongs to the group of small Hsps with anti-apoptotic and tumorigenic properties, and considered as important therapeutic targets, particularly in cancer pathology $(25,26)$. Phosphorylation by MAP kinases and AKt underlies the modulation of the apoptosis by HSPB1 (27). Our STRING results support this finding.

PTEN is another enzyme that regulates HSPB1 activity, by acting as a protein tyrosine phosphatase. Downregulation of HspB1 upregulates PTEN levels and blocks the survival pathway of cancer cells (28). In the leiomyoma, PTEN is upregulated if compared to the myometrium (29), and this may be related to the downregulation of HSPB1 in the leiomyoma.

In conclusion, the 2-DE approach is a powerful approach for comparative proteomic analysis.

In our study we identified several dysregulated chaperones associated with cell proliferation and negative regulation of apoptosis. Although our proteins have been widely validated by western blotting, the absence of an immunohistological analysis is a limitation of the present study.

These proteins take part in several key pathways involved in tumor growth. In our opinion, functional studies are essential to understand the role of these chaperones in the leiomyoma, and thus to develop inhibitors and block tumor growth.

\section{References}

1. Kim JJ and Sefton EC: The role of progesterone signaling in the pathogenesis of uterine leiomyoma. Mol Cell Endocrinol 358: 223-231, 2012.

2. Borahay MA, Al-Hendy A, Kilic GS and Boehning D: Signaling pathways in leiomyoma: Understanding pathobiology and implications for therapy. Mol Med 21: 242-256, 2015.

3. Ura B, Scrimin F, Monasta L, Radillo O and Ricci G: Association between up-regulated expression proteins and circulating steroidal hormones in leiomyoma. Med Hypotheses 85: 515, 2015.

4. Leppert PC, Catherino WH and Segars JH: A new hypothesis about the origin of uterine fibroids based on gene expression profiling with microarrays. Am J Obstet Gynecol 195: 415-420, 2006.

5. Calderwood SK and Gong J: Molecular chaperones in mammary cancer growth and breast tumor therapy. J Cell Biochem 113: 1096-1103, 2012.

6. Pratt WB and Toft DO: Regulation of signaling protein function and trafficking by the hsp90/hsp70-based chaperone machinery. Exp Biol Med (Maywood) 228: 111-133, 2003.

7. Ura B, Scrimin F, Zanconati F, Arrigoni G, Monasta L, Romano A, Banco R, Zweyer M, Milani D and Ricci G: Two-dimensional gel electrophoresis analysis of the leiomyoma interstitial fluid reveals altered protein expression with a possible involvement in pathogenesis. Oncol Rep 33: 2219-2226, 2015.

8. Ura B, Scrimin F, Franchin C, Arrigoni G, Licastro D, Monasta L and Ricci G: Identification of proteins with different abundance associated with cell migration and proliferation in leiomyoma interstitial fluid by proteomics. Oncol Lett 13: 3912-3920, 2017.

9. Ura B, Scrimin F, Arrigoni G, Athanasakis E, Aloisio M, Monasta L and Ricci G: Abnormal expression of leiomyoma cytoskeletal proteins involved in cell migration. Oncol Rep 35: 3094-3100, 2016

10. Ura B, Scrimin F, Arrigoni G, Franchin C, Monasta L and Ricci G: A proteomic approach for the identification of up-regulated proteins involved in the metabolic process of the leiomyoma. Int J Mol Sci 17: 540, 2016.

11. Carcoforo P, Ura B, Mischiati C, Squerzanti M, Lanzara V, Cervellati C, Calza R, De Laureto PP, Frare E, Portinari M, et al: Comparative proteomic analysis of ductal breast carcinoma demonstrates an altered expression of chaperonins and cytoskeletal proteins. Mol Med Rep 7: 1700-1704, 2013.

12. Mischiati C, Ura B, Roncoroni L, Elli L, Cervellati C, Squerzanti M, Conte D, Doneda L, Polverino de Laureto P, de Franceschi G, et al: Changes in protein expression in two cholangiocarcinoma cell lines undergoing formation of multicellular tumor spheroids in vitro. PLoS One 10: e0118906, 2015.

13. Ghosh JC, Dohi T, Kang BH and Altieri DC: Hsp60 regulation of tumor cell apoptosis. J Biol Chem 283: 5188-5194, 2008. 
14. Santagata S, Hu R, Lin NU, Mendillo ML, Collins LC, Hankinson SE, Schnitt SJ, Whitesell L, Tamimi RM, Lindquist S and Ince TA: High levels of nuclear heat-shock factor 1 (HSF1) are associated with poor prognosis in breast cancer. Proc Natl Acad Sci USA 108: 18378-18383, 2011.

15. Ciocca DR and Calderwood SK: Heat shock proteins in cancer: Diagnostic, prognostic, predictive, and treatment implications. Cell Stress Chaperones 10: 86-103, 2005.

16. Roh SH, Kasembeli M, Bakthavatsalam D, Chiu W and Tweardy DJ: Contribution of the type II chaperonin, TRiC/CCT, to oncogenesis. Int J Mol Sci 16: 26706-26720, 2015.

17. Blomberg A, Blomberg L, Norbeck J, Fey SJ, Larsen PM, Larsen M, Roepstorff P, Degand H, Boutry M, Posch A, et al: Interlaboratory reproducibility of yeast protein patterns analyzed by immobilized $\mathrm{pH}$ gradient two-dimensional gel electrophoresis. Electrophoresis 16: 1935-1945, 1995.

18. Lemeer S, Gholami AM, Wu Z and Kuster B: Quantitative proteome profiling of human myoma and myometrium tissue reveals kinase expression signatures with potential for therapeutic intervention. Proteomics 15: 356-364, 2015.

19. Lu YC, Weng WC and Lee H: Functional roles of calreticulin in cancer biology. Biomed Res Int 2015: 526524, 2015.

20. Schardt JA, Mueller BU and Pabst T: Activation of the unfolded protein response in human acute myeloid leukemia. Methods Enzymol 489: 227-243, 2011.

21. Roberts E, Cossigny DA and Quan GM. The role of vascular endothelial growth factor in metastatic prostate cancer to the skeleton. Prostate Cancer 2013: 418340, 2013.

22. Miyake H, Hara I, Arakawa S and Kamidono S: Stress protein GRP78 prevents apoptosis induced by calcium ionophore, ionomycin, but not by glycosylation inhibitor, tunicamycin, in human prostate cancer cells. J Cell Biochem 77: 396-408, 2000.
23. Zhang K and Kaufman RJ: Signaling the unfolded protein response from the endoplasmic reticulum. J Biol Chem 279: 25935-25938, 2004.

24. Pressinotti NC, Klocker H, Schäfer G, Luu VD, Ruschhaupt M, Kuner R, Steiner E, Poustka A, Bartsch G and Sültmann H: Differential expression of apoptotic genes PDIA3 and MAP3K5 distinguishes between low- and high-risk prostate cancer. Mol Cancer 8: 130, 2009

25. Arrigo AP, Simon S, Gibert B, Kretz-Remy C, Nivon M, Czekalla A, Guillet D, Moulin M, Diaz-Latoud C and Vicart P: Hsp27 (HspB1) and alphaB-crystallin (HspB5) as therapeutic targets. FEBS Lett 581: 3665-3674, 2007.

26. Shiota M, Bishop JL, Nip KM, Zardan A, Takeuchi A Cordonnier T, Beraldi E, Bazov J, Fazli L, Chi K, et al: Hsp27 regulates epithelial mesenchymal transition, metastasis, and circulating tumor cells in prostate cancer. Cancer Res 73: 3109-3119, 2013.

27. Arrigo AP and Gibert B: HspB1, HspB5 and HspB4 in human cancers: Potent oncogenic role of some of their client proteins. Cancers (Basel) 6: 333-365, 2014.

28. Golembieski WA, Thomas SL, Schultz CR, Yunker CK, McClung HM, Lemke N, Cazacu S, Barker T, Sage EH, Brodie C and Rempel SA: HSP27 mediates SPARC-induced changes in glioma morphology, migration, and invasion. Glia 56: 1061-1075, 2008.

29. Jeong YJ, Noh EM, Lee YR, Yu HN, Jang KY, Lee SJ, Kim J and Kim JS: 17beta-estradiol induces up-regulation of PTEN and PPARgamma in leiomyoma cells, but not in normal cells. Int J Oncol 36: 921-927, 2010. 